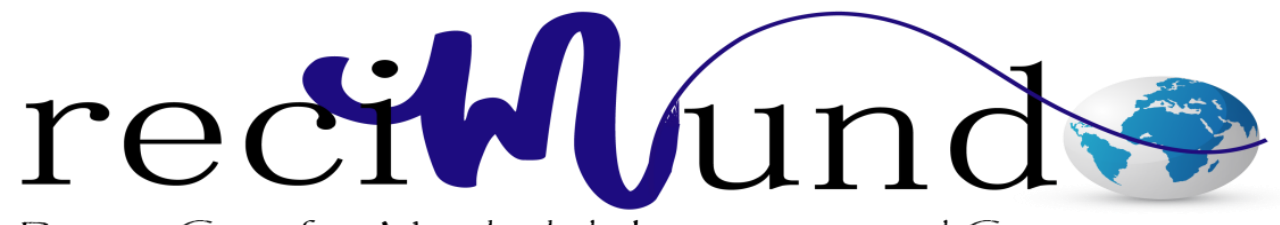

Revista Científica Mundo de la Investigación y el Conocimiento

Héctor Danilo Hugo Ullauri ${ }^{\text {a }}$ Juan Carlos Torres Espinoza ${ }^{\text {b; }}$ Víctor Eulogio Parraga Trejo ${ }^{\mathrm{c}}$

Plan estratégico para el centro de diagnóstico clínico quirúrgico - CENDIACQ; en la ciudad de Guayaquil, periodo 2015-2018

Revista Científica Mundo de la Investigación y el Conocimiento. Vol. 1 núm., 5, diciembre, 2017, pp. 129-137

DOI: $10.26820 /$ recimundo/1.5.2017.129-137

Editorial Saberes del Conocimiento
a. Universidad de Guayaquil; hector.hugou@ug.edu.ec
b. Universidad de Guayaquil; juan.torreses@ug.edu.ec
c. Universidad de Guayaquil; victor.parragat@ug.edu.ec 


\section{Plan estratégico para el centro de diagnóstico clínico quirúrgico -}

CENDIACQ; en la ciudad de Guayaquil, periodo 2015-2018

Vol. 1, núm. 5., (2017)

Héctor Danilo Hugo Ullauri; Juan Carlos Torres Espinoza; Víctor Eulogio Parraga Trejo

\section{RESUMEN}

El presente trabajo de investigación tiene como objetivo, hacer un análisis causa - efecto de la situación en general del centro de diagnóstico clínico quirúrgico (CENDIACQ) ubicado en la ciudad de Guayaquil. El diseño de la investigación es no experimental por la recolección de fuentes: campo y documental y por la perspectiva temporal del diseño: transaccional. El tipo de investigación fue cuantitativa, descriptiva y correlacional, donde se obtuvo información relevante de la organización y también de fuentes bibliográficas y electrónicas. Entre las conclusiones más importantes es que para lograr una adecuada gestión administrativa financiera del centro de diagnóstico clínico quirúrgico - CENDIACQ, se deberá contar con un plan estratégico empresarial como una herramienta de apoyo que ayude al control y direccionamiento. También es de suma importancia que se realicen anualmente herramientas de diagnóstico (Matriz FODA, Análisis Causal - Efecto, entre otros) para que se puedan tomar decisiones importantes que continúen con el óptimo funcionamiento de la organización para de esta manera evitar que amenazas o cualquier otro tipo de debilidades afecten su funcionamiento.

Palabras clave: Decisiones; matriz; análisis; organización; funcionamiento. 


\title{
Plan estratégico para el centro de diagnóstico clínico quirúrgico - CENDIACQ; en la ciudad de Guayaquil, periodo 2015-2018
}

Vol. 1, núm. 5., (2017)

Héctor Danilo Hugo Ullauri; Juan Carlos Torres Espinoza; Víctor Eulogio Parraga Trejo

\begin{abstract}
The objective of this research work is to make a cause - effect analysis of the general situation of the clinical surgical diagnosis center (CENDIACQ) located in the city of Guayaquil. The design of the research is non-experimental by the collection of sources: field and documentary and by the temporal perspective of the design: transactional. The type of research was quantitative, descriptive and correlational, where relevant information was obtained from the organization and also from bibliographic and electronic sources. Among the most important conclusions is that in order to achieve an adequate financial administrative management of the Clinical Surgical Diagnostic Center - CENDIACQ, a strategic business plan should be available as a support tool to help control and directing. It is also of utmost importance that diagnostic tools are performed annually (SWOT Matrix, Causal Analysis - Effect, among others) so that important decisions can be made that continue with the optimal functioning of the organization in order to avoid threats or any other type of weaknesses affect its functioning.
\end{abstract}

Keywords: Decisions; matrix; analysis; organization; operation. 


\section{Plan estratégico para el centro de diagnóstico clínico quirúrgico - CENDIACQ; en la ciudad de Guayaquil, periodo 2015-2018}

Vol. 1, núm. 5., (2017)

Héctor Danilo Hugo Ullauri; Juan Carlos Torres Espinoza; Víctor Eulogio Parraga Trejo

\section{Introducción.}

Hoy en día, no importa el tamaño que tenga una empresa, ni la actividad a la cual se dedique o que tanta antigüedad posea, siempre tendrá inherente la posibilidad de caer en una situación de desequilibrio financiero dado por la insolvencia y falta de liquidez; todo ello producto en muchos casos de malas políticas financieras, pero en la mayoría de las oportunidades, esta posibilidad es generada por graves errores estratégicos o acumulación de errores tanto en materia financiera como productiva, comercial y administrativa. Con el objetivo de evitar posibles situaciones de desequilibrio en las organizaciones, es un hecho que el tema de la administración se ha vuelto importante sobre todo en la época en la que nos encontramos, donde el éxito se fundamenta en la capacidad de liderazgo que tenga una empresa para afrontar los desafíos que se le presenten. (Rodriguez Lopez, Zurita Erazo, \& Pita Mateo, 2009)

Las dificultades financieras que sufren las empresas en el desarrollo de sus actividades cotidianas afectan sus relaciones con los agentes internos y externos, los cuales tienen intereses económicos para la continuidad de la empresa; asimismo, las decisiones que puedan tomar los cargos ejecutivos son afectadas debido a situaciones de insolvencia; finalmente, la economía del país es afectada si la empresa se declara en quiebra y liquida incrementando los índices de desempleo y pérdida en la actividad económica. (Cabrera \& Loaiza, 2012)

Precisamente una propuesta de reestructuración empresarial, surge como reacción a problemas de insolvencia económica que afectan en primera instancia, todo el entorno de la organización. Para lo cual es importante el diseño de propuestas y planes de acción rápida para 


\section{Plan estratégico para el centro de diagnóstico clínico quirúrgico - CENDIACQ; en la ciudad de Guayaquil, periodo 2015-2018}

Vol. 1, núm. 5., (2017)

Héctor Danilo Hugo Ullauri; Juan Carlos Torres Espinoza; Víctor Eulogio Parraga Trejo

generar los correctivos necesarios y restablecer el funcionamiento óptimo en todos sus niveles de la empresa.

Al hablar del plan estratégico de la empresa, nos estamos refiriendo al plan maestro en el que la alta dirección recoge las decisiones estratégicas corporativas que ha adoptado "hoy" ( es decir, en el momento que ha realizado la reflexión estratégica con su equipo de dirección), en referencia a lo que hará en los tres próximos años (horizonte más habitual del plan estratégico), para lograr una empresa competitiva que le permita satisfacer las expectativas de sus diferentes grupos de interés (stakeholders) (de Vicuña Ancín, 2016)

La visión de una empresa es una declaración que determina donde queremos llegar en el futuro. Una visión puede o no puede tener éxito, depende de si el resto sucede según la estrategia de la empresa. (Barrantes Rodríguez \& Tobón Vélez, 2014)

\section{Materiales y Métodos.}

La forma más común de clasificar las investigaciones es aquella que pretende ubicarse en el tiempo (según dimensión cronológica) y distingue entre la investigación de las cosas pasadas (Histórica), de las cosas del presente (Descriptiva) y de lo que puede suceder (Experimental). (Grajales, 2000)

El diseño de la investigación es no experimental por la recolección de fuentes: campo y documental y por la perspectiva temporal del diseño: transaccional.

El tipo de investigación fue cuantitativa, descriptiva y correlacional, donde se obtuvo información relevante de la organización y también de fuentes bibliográficas y electrónicas. 
Plan estratégico para el centro de diagnóstico clínico quirúrgico CENDIACQ; en la ciudad de Guayaquil, periodo 2015-2018

Vol. 1, núm. 5., (2017)

Héctor Danilo Hugo Ullauri; Juan Carlos Torres Espinoza; Víctor Eulogio Parraga Trejo

Transaccional ya que por medio de la aplicación del modelo de causa y efecto se logró comprobar por medio de sus variables las falencias que existen en la agroindustria.

Interactiva (investigación — acción, se inició con las fases exploratoria y descriptiva, pero no se limitó solo a eso, intento además proponer y cambiar). El objetivo fue sustituir un estado de cosas actual, por otro estado de cosas deseado, la obtención de datos fue una investigación participativa

Tabla $N^{\circ}$ 1: distribución de la Población y Muestras para estudio de campo

\begin{tabular}{|c|c|c|}
\hline Casos & Población & Muestra \\
\hline 1 & 8 & 8 \\
\hline 2 & 15 & 15 \\
\hline 3 & 4000 & 351 \\
\hline
\end{tabular}

Fuente: Elaboración Propia

Es habitual que en investigación cualitativa el diseño del estudio evolucione a lo largo del proyecto, por eso se dice que es emergente. En el caso del muestreo sucede lo mismo, la decisión sobre el mejor modo de obtener los datos y de quién o quiénes obtenerlos son decisiones que se toman en el campo, pues queremos reflejar la realidad y los diversos puntos de vista de los participantes, los cuales nos resultan desconocidos al iniciar el estudio. (Blanco \& Castro, 2007)

La muestra empleada para el estudio de campo fue dividida en las siguientes partes: 


\section{Plan estratégico para el centro de diagnóstico clínico quirúrgico - CENDIACQ; en la ciudad de Guayaquil, periodo 2015-2018}

Vol. 1, núm. 5., (2017)

Héctor Danilo Hugo Ullauri; Juan Carlos Torres Espinoza; Víctor Eulogio Parraga Trejo

- El 1er caso se toma el 100\% de la población interna para la aplicación del instrumento, siendo la muestra igual a 8 .

- El 2do caso el $100 \%$ de la población externa son proveedores de bienes y servicios para la aplicación del instrumento, siendo la muestra igual a 15.

- El 3er caso el 100\% de la población externa es el número de pacientes registrados para la aplicación del instrumento, siendo la muestra igual a 351 .

\section{Resultados}

Luego de la tabulación de los resultados del estudio de campo, se realizó el diagrama de causa - efecto, encontrando lo siguiente:

\section{Imagen $N^{\circ}$ 1: Diagrama de causa - efecto CENDIACQ}

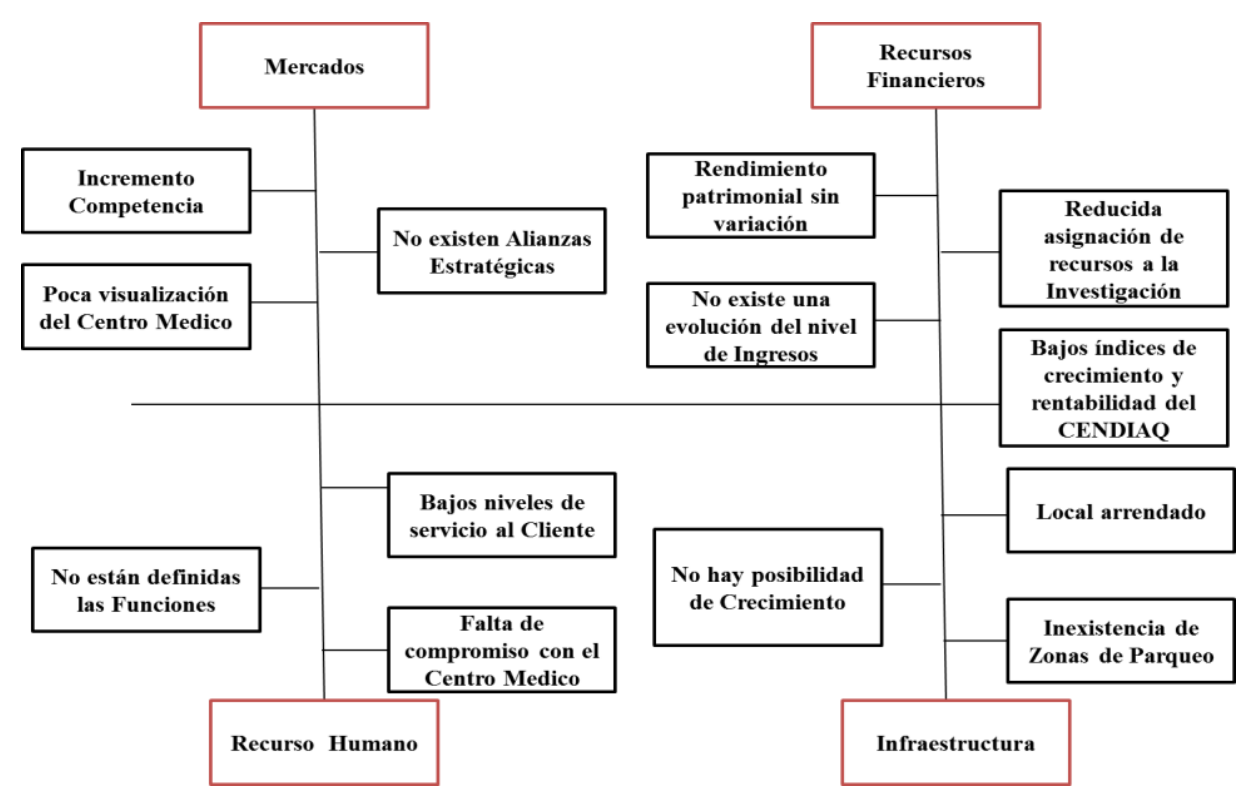

Fuente: Elaboración Propia. 
Plan estratégico para el centro de diagnóstico clínico quirúrgico CENDIACQ; en la ciudad de Guayaquil, periodo 2015-2018

Vol. 1, núm. 5., (2017)

Héctor Danilo Hugo Ullauri; Juan Carlos Torres Espinoza; Víctor Eulogio Parraga Trejo

\section{Recomendaciones}

- Luego de la elaboración del diagrama de causa - efecto, la empresa bebe abocarse a la formulación de un plan estratégico, que solucione todas las debilidades (en el área de Mercados, Recursos Humanos, Recursos Financieros e Infraestructura) en el mediano y largo plazo, para de esta manera potenciar al centro de diagnóstico clínico quirúrgico (CENDIACQ) dentro del área de la salud.

Elaboración de un plan estratégico según (Martínez Pedros et al 2007):

\section{Imagen $N^{\circ}$ 1: Elaboración de un Plan Estratégico}

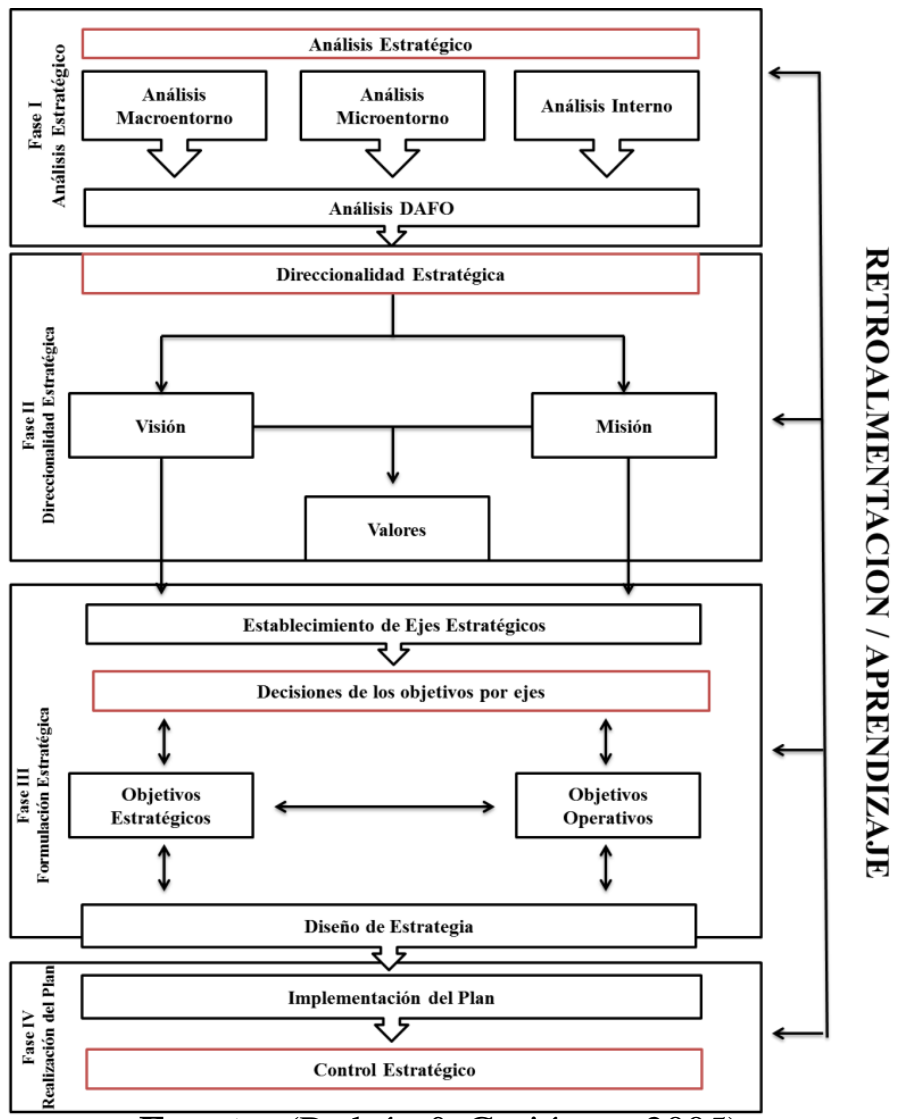

Fuente: (Pedrós \& Gutiérrez, 2005) 


\section{Plan estratégico para el centro de diagnóstico clínico quirúrgico - CENDIACQ; en la ciudad de Guayaquil, periodo 2015-2018}

Vol. 1, núm. 5., (2017)

Héctor Danilo Hugo Ullauri; Juan Carlos Torres Espinoza; Víctor Eulogio Parraga Trejo

\section{Conclusiones}

El centro de diagnóstico clínico quirúrgico (CENDIACQ), debe realizar el estudio de su mercado y del entorno interno para de esta manera tener la información precisa de todos aquellos elementos que los puedan debilitar o amenazar. Luego de recabar toda la información deben diseñarse las estrategias para afrontar todas las debilidades, las cuales deben estar asentadas por escrito (fichas, planes, matrices, cuadros de mando) para que puedan ser evaluadas continuamente y hacer los correctivos necesarios, si fuera el caso.

\section{Bibliografía}

Barrantes Rodríguez, A., \& Tobón Vélez, J. (2014). Biblioteca Digital Universidad de San Buenaventura. Recuperado el 10 de 12 de 2017, de http://bibliotecadigital.usb.edu.co/handle/10819/2274

Blanco, M. C., \& Castro, A. (2007). El muestreo en la investigación cualitativa.

Cabrera, A., \& Loaiza, X. (2012). Repositorio Universidad Nacional de Loja. Recuperado el 10 de 12 de 2017, de http://dspace.unl.edu.ec/jspui/handle/123456789/2013

de Vicuña Ancín, J. (2016). El plan de marketing en la PYME. ESIC.

Grajales, T. (2000). Tipos de investigación. On line. Recuperado el 10 de 12 de 2017, de http://tgrajales.net/investipos.pdf

Pedrós, D., \& Gutiérrez, A. (2005). La elaboración del plan estratégico y su implantación a través del cuadro de mando integral. Díaz de Santos.

Rodriguez Lopez, B., Zurita Erazo, S., \& Pita Mateo, M. (27 de 02 de 2009). DSpace Repository. $\begin{array}{lllllll}\text { Recuperado el } & 10 & \text { de } & 12 & \text { de } & 2017, & \text { de }\end{array}$ http://www.dspace.espol.edu.ec/xmlui/handle/123456789/1347 